\title{
KrkNLO IN HERWIG $7^{*}$
}

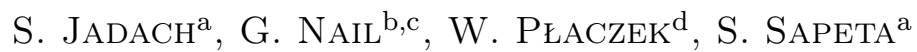 \\ A. SIÓDMOK ${ }^{\mathrm{a}}$, M. SKRZYPEK ${ }^{\mathrm{a}}$
}

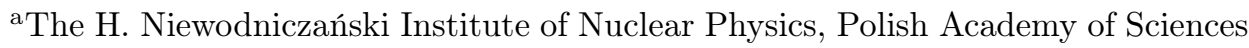
Radzikowskiego 152, 31-342 Kraków, Poland

${ }^{\mathrm{b}}$ Particle Physics Group, School of Physics and Astronomy

University of Manchester, Oxford Road, Manchester, M13 9PL, United Kingdom

${ }^{\mathrm{c}}$ Institute for Theoretical Physics, Karlsruhe Institute of Technology

Wolfgang-Gaede-Strasse 1, 76131 Karlsruhe, Germany

${ }^{\mathrm{d}}$ The M. Smoluchowski Institute of Physics, Jagiellonian University

Łojasiewicza 11, 30-348 Kraków, Poland

(Received April 21, 2017)

We validate and present new results from the first implementation of the KrkNLO method within the Herwig 7 event generator. In this work, we present numerical results for the Drell-Yan process, and Higgs-boson production via gluon fusion. The Drell-Yan process is used to validate this new implementation against the previous version in Sherpa, as well as to compare the recently introduced complete MC-scheme parton distribution functions to previous results. We present the first results of the KrkNLO method for Higgs production via gluon fusion at the LHC, and compare them to MCONLO and POWHEG predictions from Herwig 7, as well as results from HNNLO and $\mathrm{HqT}$.

DOI:10.5506/APhysPolB.48.1121

\section{Introduction}

The discovery of the Higgs boson at the Large Hadron Collider (LHC) $[1,2]$ was an important milestone in the exploration of the electroweak sector of the Standard Model (SM). The measurement of the mass of this new particle turns the Higgs sector into a fully predictive theory, and, therefore, we are in a position to preform rigorous tests of our understanding of these fundamental interactions. Though, this is only possible if we possess precise theoretical predictions.

* Presented by G. Nail at the Cracow Epiphany Conference "Particle Theory Meets the First Data from LHC Run 2", Kraków, Poland, January 9-12, 2017. 
The production of Higgs bosons at hadron colliders is dominated by gluon fusion; a channel known to exhibit slow convergence. At the energies probed by the LHC, the corrections due to next-to-leading (NLO) contributions are as large as $70 \%$, and the next-to-next-to-leading order (NNLO) results add a further $30 \%$ [3-5]. The theoretical uncertainties at NNLO, obtained by the standard factorization and renormalization scale variations, are estimated to be around 10\%, which is comparable to the experimental accuracy of Run 1. This large uncertainty has motivated the calculation of the N3LO contributions to this production mode [6].

Aside from measuring the inclusive cross section, one is also interested in measuring the Higgs properties in more differential observables, such as transverse momentum and rapidity. A significant amount of work has gone into such calculations, for example: small- $_{\mathrm{T}}$ coordinate-space resummation up to NNLL+NLO accuracy [7] and in momentum space up to NNLL+NNLO accuracy [8] within the framework of analytic resummation; as well as in SCET up to NNLL+NLO accuracy [9]. These distributions have also been studied in the context of fixed-order NLO results matched to parton shower $(\mathrm{NLO}+\mathrm{PS})$. The next major challenge is the combination of NNLO corrections with a NLO parton shower (NNLO+NLOPS).

In this work, we outline the KrkNLO method, and the ingredients necessary to implement it within the Herwig 7 program. We validate this new implementation against the results of a previous implementation and present new results concerning the recently introduced complete MC-scheme parton distribution functions (PDFs), as well as new NLO+PS predictions for various differential distributions in Higgs production via gluon-fusion computed using the KrkNLO approach. The results are presented within the Herwig 7 Parton-Shower Monte Carlo [10, 11]. The advantage of this method is its simplicity; the NLO corrections are implemented using a simple, positive, multiplicative weight in combination with pre-calculated MC-scheme PDFs. This work forms a platform for the future developments as the simplifications present at NLO will be valuable in obtaining NNLO+NLOPS accuracy.

\section{KrkNLO}

The KrkNLO method for matching fixed-order NLO QCD corrections to LO parton-shower Monte Carlo generators was first introduced in Ref. [12] for the Drell-Yan (DY) process; this work was also presented in Ref. [13]. This followed the initial study of Refs. $[14,15]$ which used a dedicated toymodel parton-shower generator, with gluonstrahlung from quarks only, for DY and deep inelastic electron-proton scattering (DIS). 
The first realistic numerical results of the KrkNLO method, based on an implementation in the Sherpa [16] PSMC, were presented in Ref. [12] for the DY process. In this work, the total cross section and distributions of the $Z / \gamma^{*}$ transverse momentum and rapidity were compared to the fixedorder NLO prediction of MCFM [17], and the NLO+PS matching methods of MC@NLO [18, 19] and POWHEG [20, 21], as well as fixed-order NNLO calculations from DYNNLO [22]. This predictions of KrkNLO showed satisfactory agreement with the other NLO calculations.

In Ref. [12], the concept of the Monte Carlo (MC) factorization scheme was introduced as a necessary ingredient of the KrkNLO method; this was further developed in Refs. [23, 24]. There are two variants of PDF available in the MC-scheme: $\mathrm{MC}_{\mathrm{DY}} \mathrm{PDFs}$, and MC PDFs. In the former, only the quark PDFs are transformed from the $\overline{\mathrm{MS}}$-scheme to the MC-scheme, leaving the gluon PDF unchanged, and in the latter, the gluon PDF is also transformed to the MC-scheme. For the DY process at NLO, it is sufficient to use the $\mathrm{MC}_{\mathrm{DY}} \mathrm{PDF}$, however, Higgs production via gluon fusion requires the complete MC PDFs due to the presence of initial-state gluons at LO.

The KrkNLO method requires three key components: PDFs defined in the MC-scheme, an LO PSMC that covers the entire NLO phase-space, and the KrkNLO multiplicative weights. For an NLO subprocess

$$
a\left(p_{1}\right)+b\left(p_{2}\right) \longrightarrow X+c(k),
$$

with partons $a, b, c$ and $X=H, Z$, shown in Fig. 1, the kinematics is parametrised by the Sudakov variables that are defined as follows:

$$
\alpha=\frac{p_{1} \cdot k}{p_{1} \cdot p_{2}}, \quad \beta=\frac{p_{2} \cdot k}{p_{1} \cdot p_{2}}, \quad \alpha+\beta=1-z \leq 1 .
$$

The $\alpha, \beta$ used are those which are understood to refer the parton with the maximum $k_{\mathrm{T}}$, in the Herwig $7 k_{\mathrm{T}}$-ordered dipole shower this is the first emission. The multiplicative weights rescale the parton-shower emission to the full matrix element by

$$
W_{\mathrm{R}}=\left|\mathcal{M}_{a b \rightarrow X c}^{\mathrm{NLO}}\right|^{2} /\left|\mathcal{M}_{a b \rightarrow X c}^{\mathrm{MC}}\right|^{2},
$$

where $\mathcal{M}^{\mathrm{NLO}}$ is the full NLO matrix-element, and $\mathcal{M}^{\mathrm{MC}}$ is the shower approximation. Finally, there is also a weight associated with virtual + soft corrections. In order to generate KrkNLO events, it is necessary to first generate events at LO using the MC-scheme PDFs, and shower them. Then, apply the KrkNLO real weight to the first emission and the virtual+soft weight to the whole event. The required weights are outlined below. 


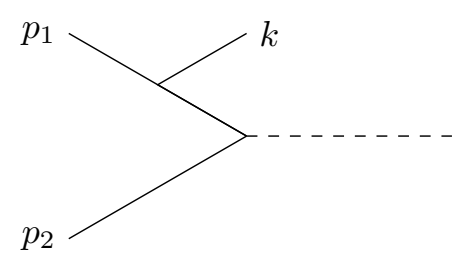

Fig. 1. The kinematic set-up for KrkNLO method. The incoming partons have momementa $p_{1}$, and $p_{2}$, and the real emission has momentum $k$.

\subsection{Drell-Yan}

For the DY process, there are two NLO subprocesses that need to be corrected: $q \bar{q}$ and $q g$. The real weights for these subprocesses are:

(a) $q+\bar{q} \longrightarrow Z+g$

$$
W_{\mathrm{R}}^{q \bar{q}}(\alpha, \beta)=1-\frac{2 \alpha \beta}{1+z^{2}}
$$

(b) $q+g \longrightarrow Z+q$

$$
W_{\mathrm{R}}^{q g}(\alpha, \beta)=1+\frac{\beta(\beta+2 z)}{z^{2}+(1-z)^{2}}, \quad W_{\mathrm{R}}^{q g}(\alpha, \beta)=W_{\mathrm{R}}^{g q}(\beta, \alpha) .
$$

The virtual + soft weights, $W_{\mathrm{VS}}=1+\Delta_{\mathrm{VS}}$ for the two channels are:

$$
\Delta_{\mathrm{VS}}^{q \bar{q}}=\frac{\alpha_{\mathrm{S}}}{2 \pi} C_{\mathrm{F}}\left(\frac{4}{3} \pi^{2}+\frac{1}{2}\right), \quad \Delta_{\mathrm{VS}}^{q g}=0 .
$$

\subsection{Higgs production via gluon fusion}

For Higgs-production via gluon fusion, there are three NLO subprocesses that need to be corrected: $g g, g q$ and $q \bar{q}$. However, the $q \bar{q}$ initiated state cannot be generated via backward evolution, and must be added as an additional tree-level LO matrix element. The real weights for the remaining subprocesses are:

(a) $g+g \longrightarrow H+g$

$$
W_{\mathrm{R}}^{g g}(\alpha, \beta)=\frac{1+z^{4}+\alpha^{4}+\beta^{4}}{1+z^{4}+(1-z)^{4}},
$$

(b) $g+q \longrightarrow H+q$

$$
W_{\mathrm{R}}^{g q}(\alpha, \beta)=\frac{1+\beta^{2}}{1+(1-z)^{2}}, \quad W_{\mathrm{R}}^{q g}(\alpha, \beta)=W_{\mathrm{R}}^{g q}(\beta, \alpha) .
$$


The virtual + soft weights, $W_{\mathrm{VS}}=1+\Delta_{\mathrm{VS}}$, for these two channels are:

$$
\Delta_{\mathrm{VS}}^{g g}=\frac{\alpha_{\mathrm{s}}}{2 \pi} C_{\mathrm{A}}\left(\frac{4 \pi^{2}}{3}+\frac{473}{36}-\frac{59}{18} \frac{T_{f}}{C_{\mathrm{A}}}\right), \quad \Delta_{\mathrm{VS}}^{g q}=0 .
$$

These weights are calculated for the large top mass approximation, that has $m_{t} \rightarrow \infty$ and $m_{q \neq t}=0$.

\section{Numerical results}

The numerical results in this section for proton-proton collisions at the LHC were performed at an energy of $\sqrt{s}=8 \mathrm{TeV}$ and with the following Standard Model parameters:

$$
\begin{array}{rlrl}
M_{Z} & =91.1876 \mathrm{GeV}, & \Gamma_{Z} & =2.4952 \mathrm{GeV}, \\
M_{W} & =80.4030 \mathrm{GeV}, & \Gamma_{W} & =2.1240 \mathrm{GeV}, \\
M_{H} & =126.0 \mathrm{GeV}, & M_{t} & =173.2 \mathrm{GeV}, \\
G_{\mu} & =1.16637 \times 10^{-5} \mathrm{GeV}^{-2} .
\end{array}
$$

The $G_{\mu}$-scheme [25] was used for the electroweak sector. The factorization and renormalization scales were set to $\mu_{\mathrm{F}}=\mu_{\mathrm{R}}=M$, where $M$ is the mass of the $Z$ or Higgs boson for the relevant process. The PDF and $\alpha_{\mathrm{s}}$ setting is detailed in the relevant subsection.

\subsection{Drell-Yan}

The results for the KrkNLO implementation built on top of Sherpa for the Drell-Yan process have been presented in Ref. [16]; the results for the new implementation in Herwig 7 will follow the same set-up and will be compared with the previous results for validation. These results use the MSTW2008LO parton distribution functions [26], which have $\alpha_{\mathrm{s}}\left(M_{Z}^{2}\right)=0.13938690$. The analysis imposes a cut on the invariant mass of the final-state lepton pair of

$$
50 \mathrm{GeV}<m_{\overline{l l}}<150 \mathrm{GeV} .
$$

As a foundation for the validation, we first compare the LO results of the total cross section, Table I, from MCFM, Sherpa and Herwig 7. These show a good agreement within statistical errors. When comparing distributions of the final-state lepton pair, Fig. 1 of Ref. [27], we also see a good agreement. In the case of the $Z p_{\mathrm{T}}$ spectrum, differences between Sherpa and Herwig 7 arise from different treatments of intrinsic- $k_{\mathrm{T}}$ and the soft-parton limit, where the latter emerges from the different ordering variables present in the two programs. 
TABLE I

Values of the total cross section, with statistical errors, for the Drell-Yan process comparing LO fixed order results from MCFM, and LO results from Sherpa and Herwig 7 using the $\overline{\mathrm{MS}}$-scheme PDFs.

\begin{tabular}{c|ccc}
\hline \hline & MCFM & Sherpa & Herwig 7 \\
\hline$\sigma_{\text {tot }}[\mathrm{pb}]$ & $936.9(1)$ & $937.2(2)$ & $936.6(2)$
\end{tabular}

To validate the implementation in Herwig 7, we now compare the KrkNLO results to the previous implementation in Sherpa. We simulate both the $q \bar{q}$ and $q g$ NLO channels of the DY process. In this set-up, the scale used for the hard-real NLO correction is the evolution variable $q^{2}, \alpha_{\mathrm{s}}\left(q^{2}\right)$, and for the virtual + soft real correction, the scale used is $M_{Z}^{2}, \alpha_{\mathrm{s}}\left(M_{Z}^{2}\right)$. Both set-ups use the $\mathrm{MC}_{\mathrm{DY}}$-scheme variants of the PDFs. Comparing the total cross sections, detailed in Table II, we see that both KrkNLO implementations give cross sections that agree at the per-mille level with the small residual dependency owing the aforementioned differences in the low- $p_{\mathrm{T}}$ region; this can be seen in Fig. 2 of Ref. [27]. With this agreement, we are able to validate our implementation of the KrkNLO method in Herwig 7.

TABLE II

Values of the total cross section, with statistical errors for the Drell-Yan process comparing NLO fixed order results from MCFM ( $\overline{M S}$ PDFs), and KrkNLO (MCDY PDFs) results from Sherpa and Herwig 7.

\begin{tabular}{c|c|cc}
\hline \hline & & \multicolumn{2}{|c}{ KrkNLO } \\
\cline { 3 - 4 } & \multirow{2}{*}{ MCFM } & Sherpa & Herwig 7 \\
\hline$\sigma_{\text {tot }}[\mathrm{pb}]$ & $1086.5(1)$ & $1045.2(3)$ & $1046.5(2)$
\end{tabular}

The first new results using this implementation compare the predictions from two variants of the MC-scheme: $\mathrm{MC}_{\mathrm{DY}} \mathrm{PDFs}$, first introduced in Ref. [12], and the complete MC PDFs, introduced in Ref. [23]. The difference between the $\mathrm{MC}_{\mathrm{DY}} \mathrm{PDF}$ and $\mathrm{MC} \mathrm{PDF}$ is that the former transforms only the quark $\overline{\mathrm{MS}} \mathrm{PDF}$ to the MC-scheme, leaving the gluon PDF unchanged, whereas the latter transforms also the gluon PDF to the MC-scheme. For the DY process, it is sufficient to use the $\mathrm{MC}_{\mathrm{DY}} \mathrm{PDF}$ as the contribution from the transformed gluon PDFs is a beyond-NLO effect. The difference in the total cross section, shown in Table III, between the two variants of the MC-scheme is rather modest, at $\sim 2 \%$, and is well within the uncertainty of NLO calculations. The differences compared to the cross section from an $\overline{\mathrm{MS}}$ PDFs using MCFM is at a level of $4-6 \%$, and is also within the NLO 
accuracy. Large differences in kinematic distributions between the two MC variants occur in regions that are sensitive to the soft-gluon contribution, and hence the gluon PDF; this can be seen in Fig. 3 of Ref. [27].

TABLE III

Values of the total cross section, with statistical errors for the Drell-Yan process from KrkNLO implemented in Herwig 7 with two variants of MC-scheme PDFs compared with the fixed-order NLO result from MCFM.

\begin{tabular}{c|c|cc}
\hline \hline & & \multicolumn{2}{|c}{ KrkNLO } \\
\cline { 3 - 4 } & MCFM: $\overline{\mathrm{MS}}$ PDFs & $\mathrm{MC}_{\mathrm{DY}}$ PDFs & MC PDFs \\
\hline$\sigma_{\text {tot }}[\mathrm{pb}]$ & $1086.5(1)$ & $1046.5(2)$ & $1022.3(2)$
\end{tabular}

\subsection{Higgs production via gluon fusion}

In this section, we present results for Higgs-boson production in gluongluon fusion at the LHC with the KrkNLO method implemented in Herwig 7. These results are compared to predictions from other NLO+PS matching approaches, namely MCONLO[19] and POWHEG [21] as implemented in Herwig 7. Additionally, we compare to fixed-order calculations at NLO and NNLO from HNNLO [5] and an NNLL+NNLO calculation from HqT [7, 28]. The simulations are performed using the CT10nlo PDF set [29] which has $\alpha_{\mathrm{S}}\left(M_{Z}^{2}\right)=0.118$. There are no cuts applied for Higgs production, and, for simplicity, we set the Higgs boson to be stable.

As a starting point, we compare the total cross section for the LO results between Herwig 7 and HNNLO as a baseline for the NLO results. The total cross sections, shown in Table IV, are in a very good agreement between these programs.

TABLE IV

Values of the total cross section with statistical errors (in brackets) at LO level for Higgs production in gluon-gluon fusion at the LHC for the $\overline{\mathrm{MS}}$ CT10nlo $\left(\alpha_{\mathrm{s}}=\right.$ 0.118) PDFs from HNNLO and Herwig 7 (fixed order).

\begin{tabular}{c|cc}
\hline \hline & HNNLO@LO & Herwig 7 \\
\hline$\sigma_{\text {tot }}[\mathrm{pb}]$ & $5.565(1)$ & $5.564(1)$
\end{tabular}

We present the results from KrkNLO and compare them to those of the MCQNLO and POWHEG methods implemented in Herwig 7 as well as the NLO and NNLO results from HNNLO and a further result from HqT. The KrkNLO set-up uses $\alpha_{\mathrm{s}}\left(q^{2}\right)$ for the hard-real corrections and $\alpha_{\mathrm{s}}\left(M_{H}^{2}\right)$ for the 
virtual + soft real corrections. We show two variants of the POWHEG method. The first, POWHEG (Default), is the default set-up in Herwig 7 and restricts the transverse momentum of parton-shower emissions to be less than the factorization scale, as is done in the MCONLO set-up, which follows the initial work of Ref. [18]. The second, POWHEG (Original), is closer to its original implementation [21] which has no such restriction. This amounts to POWHEG (Default) generating both $S$ - and $H$-events, with POWHEG (Original) only generating $S$-events.

The values of the total cross sections are presented in Tables V-VI. As expected, the MC@NLO and both POWHEG results are in a very good agreement. The KrkNLO result is slightly higher than the other matching approaches, which can be explained by beyond-NLO contributions that are partially accounted for in the KrkNLO method. The cross sections are above the value predicted by the fixed-order HNNLO result, however, they are well within the, rather large, uncertainty band obtained by varying the factorization and renormalization scales by the typical factors of $1 / 2$ and 2 around the central value. Furthermore, the NNLO uncertainty band is also rather large, at around $10 \%$, and does not overlap with the NLO uncertainty band. This can be seen in Fig. 4 of Ref. [27].

TABLE V

Values of the total cross section, with statistical errors for Higgs-boson production in gluon-gluon fusion at the LHC for KrkNLO, MC@NLO and POWHEG as calculated by Herwig 7 .

\begin{tabular}{c|c|cc|c}
\hline \hline & & \multicolumn{2}{|c|}{ POWHEG } & \\
\cline { 3 - 4 } & \multirow{2}{*}{ MC@NLO } & Default & Original & KrkNLO \\
\hline$\sigma_{\text {tot }}[\mathrm{pb}]$ & $12.700(2)$ & $12.699(2)$ & $12.697(2)$ & $12.939(2)$
\end{tabular}

TABLE VI

Values of the total cross section, with statistical errors for Higgs-boson production in gluon-gluon fusion at the LHC from NLO and NNLO simulations from HNNLO.

\begin{tabular}{c|cc}
\hline \hline & \multicolumn{2}{|c}{ HNNLO } \\
\cline { 2 - 3 } & NLO & NNLO \\
\hline$\sigma_{\text {tot }}[\mathrm{pb}]$ & $12.640(1)$ & $17.063(15)$
\end{tabular}

In Fig. 2, we present distributions of the transverse momentum, $p_{\mathrm{T}}^{H}$, and rapidity, $y^{H}$, of the Higgs boson and compare the results of KrkNLO to MC@NLO and POWHEG. These agree to within a few per-cent in the $p_{\mathrm{T}}^{H}$ range from $\sim 5 \mathrm{GeV}$ to $\sim 100 \mathrm{GeV}$ and for the rapidity range $\left|y^{H}\right|<3$. 
In the region of $p_{\mathrm{T}}^{H}>M_{H}$, there are larger differences between KrkNLO and MC@NLO/POWHEG (Default), which is a consequence of the restricted emission phase-space; one can see the effect of this restriction from the sharp drop around $M_{H}$. POWHEG (Original) shows the same behaviour as KrkNLO which has no such restriction. However, such differences are acceptable within the NLO approximation.
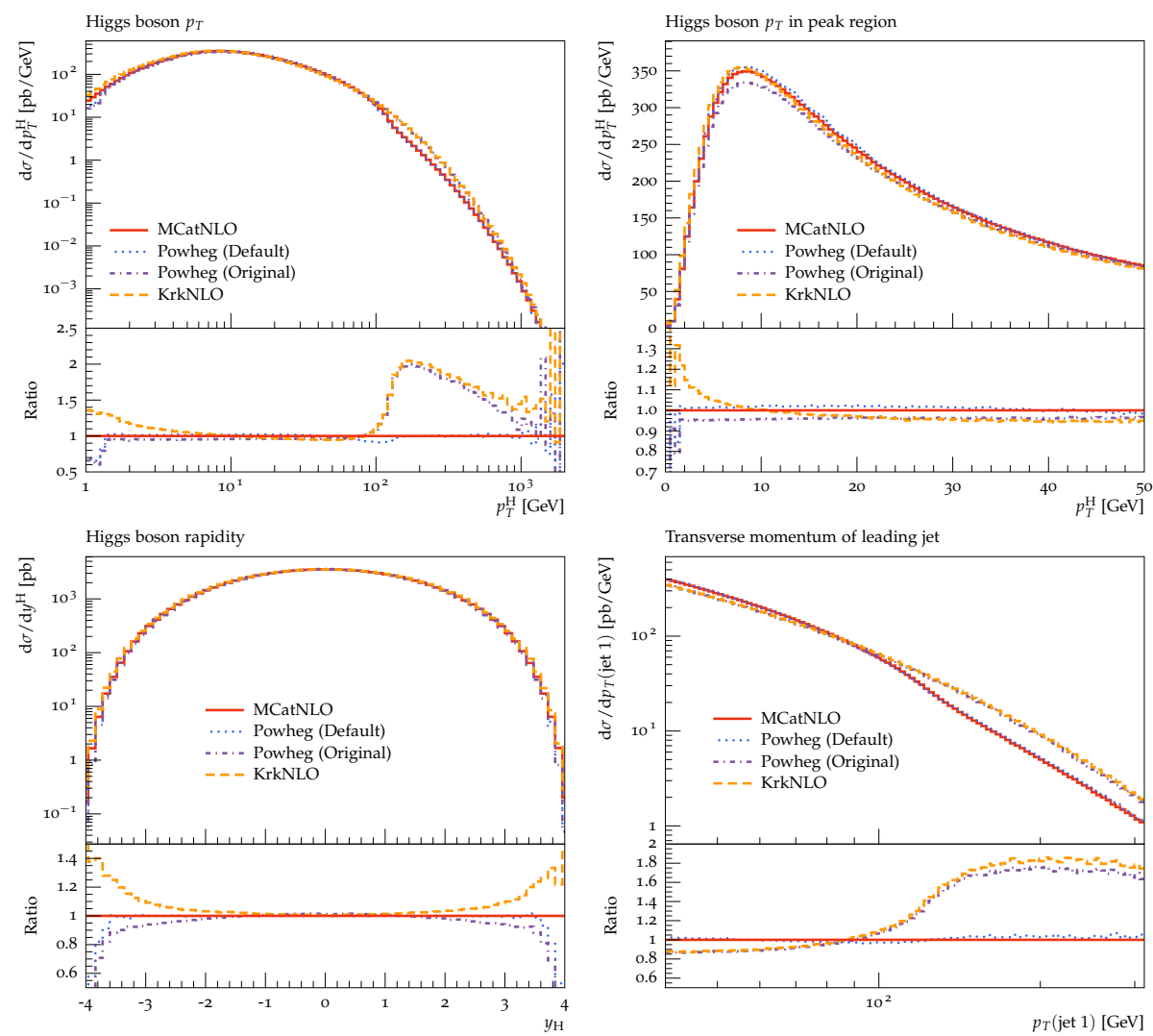

Fig. 2. Comparisons of the Higgs-boson transverse momentum and rapidity distributions from the KrkNLO, MC@NLO and POWHEG methods implemented in Herwig 7 for the Higgs-boson production in gluon-gluon fusion at the LHC.

Figure 3 shows the KrkNLO, MC@NLO and POWHEG results for the transverse momentum of the Higgs boson compared to results from HNNLO and $\mathrm{HqT}$. The error bands for HNNLO were obtained by varying the factorization and renormalization scales by the typical factors of $1 / 2$ and 2 around $M_{H}$ as an estimate of the uncertainty from neglected higher orders. The HqT error band is obtained similarly, but also contains a variation of the resummation scale by factors of $1 / 2$ and 2 around the central scale of $M_{H} / 2$. 
The HNNLO comparison (left plot) is shown relative to the NLO distribution from HNNLO, and the HqT comparison (right plot) is shown relative to the NNLO distribution from HqT. In the HNNLO comparison, we see that the $\mathrm{NLO}+\mathrm{PS}$ curves all rise along with the NNLO as low $p_{\mathrm{T}}^{H}$, but that at high $p_{\mathrm{T}}^{H}$, the MC@NLO and POWHEG (Default), by construction, converge to the NLO result. The KrkNLO and POWHEG (Original) are close to the NNLO predictions, but at very large $p_{\mathrm{T}}^{H}$, they are marginally harder. In the $\mathrm{HqT}$ comparison, the $\mathrm{NLO}+\mathrm{PS}$ results give similar results up to roughly $80 \mathrm{GeV}$, but we notice that the $\mathrm{HqT}$ resummed result peaks towards lower values of $p_{\mathrm{T}}^{H}$.
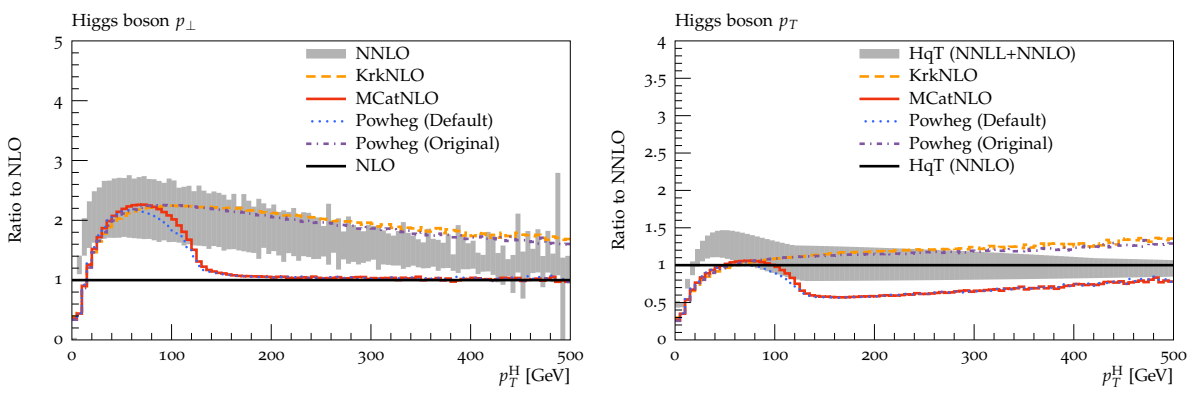

Fig. 3. The Higgs-boson transverse momentum distributions from KrkNLO, POWHEG and MC@NLO. The left plot compares our results with the fixed-order NNLO result from the HNNLO program and are shown relative to the NLO results from HNNLO. The right plot shows our results in comparison to $\mathrm{HqT}$, these are shown relative to the HqT NNLO prediction. The content of the error bands is described in the main text.

\section{Conclusion}

In this work, we have presented numerical results for the implementation of KrkNLO method in Herwig 7. We have used the Drell-Yan process to validate the implementation against a previous one in Sherpa, and to explore the differences between the $\mathrm{MC}_{\mathrm{DY}} \mathrm{PDF}$ and the recently introduced complete MC PDFs. The difference between these variants was found to be $\sim 2 \%$. The main body of the work concerned Higgs production via gluon fusion where we presented total cross sections and distributions of rapidity and transverse momentum. The results of the KrkNLO method were compared to other matching approaches: MC@NLO and POWHEG. A good agreement was found between these methods, with KrkNLO showing a very good agreement with the POWHEG (Original) option.

The KrkNLO project will be available at https://krknlo .hepforge.org, where its development will continue. The site will host the MC-scheme PDFs, sample input cards and other relevant codes. 
Future work will investigate more Drell-Yan processes, involving both neutral $\left(Z / \gamma^{*}\right)$ and charged $\left(W^{ \pm}\right)$modes in the presence of experimental cuts, and a focus on leptonic observables. This necessitates the inclusion of spin correlations in the correcting weights for vector-boson decay products.

Beyond this, the natural extension of the KrkNLO approach is to attempt $\mathrm{NNLO}+\mathrm{NLOPS}$, where the simplifications realised by this approach will, in our opinion, be instrumental in achieving this ambitious aim.

\section{REFERENCES}

[1] G. Aad et al. [ATLAS Collaboration], Phys. Lett. B 716, 1 (2012) [arXiv: 1207.7214 [hep-ex]].

[2] S. Chatrchyan et al. [CMS Collaboration], Phys. Lett. B 716, 30 (2012) [arXiv:1207.7235 [hep-ex]].

[3] R.V. Harlander, W.B. Kilgore, Phys. Rev. Lett. 88, 201801 (2002) [arXiv:hep-ph/0201206].

[4] C. Anastasiou, K. Melnikov, Nucl. Phys. B 646, 220 (2002) [arXiv:hep-ph/0207004].

[5] S. Catani, M. Grazzini, Phys. Rev. Lett. 98, 222002 (2007) [arXiv:hep-ph/0703012].

[6] C. Anastasiou et al., Phys. Rev. Lett. 114, 212001 (2015) [arXiv:1503.06056 [hep-ph]].

[7] D. de Florian, G. Ferrera, M. Grazzini, D. Tommasini, J. High Energy Phys. 11, 064 (2011) [arXiv:1109.2109 [hep-ph]].

[8] P.F. Monni, E. Re, P. Torrielli, Phys. Rev. Lett. 116, 242001 (2016) [arXiv:1604.02191 [hep-ph]].

[9] T. Becher, M. Neubert, D. Wilhelm, J. High Energy Phys. 1305, 110 (2013) [arXiv:1212.2621 [hep-ph]].

[10] M. Bahr et al., Eur. Phys. J. C 58, 639 (2008) [arXiv:0803.0883 [hep-ph]].

[11] J. Bellm et al., Eur. Phys. J. C 76, 196 (2016) [arXiv:1512.01178 [hep-ph]].

[12] S. Jadach et al., J. High Energy Phys. 1510, 052 (2015) [arXiv:1503.06849 [hep-ph]].

[13] S. Jadach et al., Acta Phys. Pol. B 46, 2089 (2015).

[14] S. Jadach et al., Phys. Rev. D 87, 034029 (2013) [arXiv:1103.5015 [hep-ph]].

[15] S. Jadach et al., Acta Phys. Pol. B 43, 2067 (2012) [arXiv:1209.4291 [hep-ph]].

[16] T. Gleisberg et al., J. High Energy Phys. 0902, 007 (2009) [arXiv:0811.4622 [hep-ph]]. 
[17] J.M. Campbell, R.K. Ellis, Phys. Rev. D D60, 113006 (1999) [arXiv:hep-ph/9905386]; http://mcfm.fnal.gov

[18] S. Frixione, B.R. Webber, J. High Energy Phys. 0206, 029 (2002) [arXiv:hep-ph/0204244].

[19] S. Frixione, B.R. Webber, arXiv:hep-ph/0601192.

[20] P. Nason, J. High Energy Phys. 0411, 040 (2004) [arXiv:hep-ph/0409146].

[21] S. Frixione, P. Nason, C. Oleari, J. High Energy Phys. 0711, 070 (2007) [arXiv:0709.2092 [hep-ph]].

[22] S. Catani et al., Phys. Rev. Lett. 103, 082001 (2009) [arXiv:0903.2120 [hep-ph]].

[23] S. Jadach et al., arXiv:1606.00355 [hep-ph].

[24] S. Jadach et al., PoS LL2016, 020 (2016) [arXiv:1607.00919 [hep-ph]].

[25] G. Altarelli, M.L. Mangano, in: proceedings of the Workshop on Standard Model Physics (and More) at the LHC, May 9, 2000, Yellow Report, CERN 2000-04.

[26] A.D. Martin, W.J. Stirling, R.S. Thorne, G. Watt, Eur. Phys. J. C 63, 189 (2009) [arXiv:0901.0002 [hep-ph]].

[27] S. Jadach et al., Eur. Phys. J. C 77, 164 (2017) [arXiv: 1607.06799 [hep-ph]].

[28] G. Bozzi, S. Catani, D. de Florian, M. Grazzini, Nucl. Phys. B 737, 73 (2006) [arXiv:hep-ph/0508068].

[29] H.-L. Lai et al., Phys. Rev. D 82, 074024 (2010) [arXiv:1007.2241 [hep-ph]]. 